\title{
Insulator String Cleaning Robot
}

\author{
Changfeng Li \\ School of NORTH CHINA ELECTRIC POWER University(Baoding), BaoDing 071000, China
}

az751428870@126.com

Keywords: Insulator, Cleaning, Robot.

\begin{abstract}
China's power system continues to develop. More and more high-voltage substation have been built. The safe operation of power systems is more and more important. Substation insulators susceptible to the natural environment and other factors, there are the formation of pollution accumulation on the surface. It causes Prone to corona, pollution flash wear and other accidents, resulting in short circuit, substation trips, large area power outages and other major accidents. As a result of manual cleaning need to power outage, with a little bit of water caused by secondary pollution, this paper studied insulator string cleaning robot to solve this problem. A brief introduction to the domestic and foreign insulator cleaning related research, for the insulator cleaning needs to determine the design direction. Analysis of pillar insulator specifications, to determine the design for vertical lift, covered cleaning. According to the design and design requirements to build a simple model, the use of STM32 microcontroller with Keil5 programming software to complete the model movement, cleaning and other action control. Through the study of the model, verify the practicality of the insulator string cleaning robot, and promote the automation and intelligentization of the transformer insulation of the substation.
\end{abstract}

\section{Introduction}

\subsection{Background and Significance}

With the development of society and the improvement of industrial level, substation and other high-voltage transmission sites continue to build, greatly improving the efficiency of national electricity, while the safe operation of power systems become a crucial issue. Among them, the substation insulator string is often affected by the complexity of the natural environment factors, in the surface of the formation of difficult to clean the dirt, resulting in flash lightning or corona and other accidents, resulting in substation trips and large area power outages, serious even cause casualties . Not only to reduce production efficiency and power system to the safe operation of the larger risks.

At present, most of China's substation on the insulator string anti-pollution flash work mainly to take two methods of $\mathrm{Tu}$ and sweep. Coated as protective measures, such as strengthening the insulation configuration, the installation of auxiliary umbrella skirt, brushing the surface of the insulator silicone oil and silicone grease, coated with anti-pollution flash organic paint and so on. Sweep is clean measures, there are major power manual scrub, charged water red, with electric blow, mechanical dry cleaning and so on.

Therefore, Compared with the current mainstream cleaning method, we propose substation insulator string cleaning robot design,can save manpower and material resources, and will not produce secondary pollution, not only to ensure the safe operation of the power system, more in line with the concept of modern environment friendly, effectively solve the current substation insulator string cleaning.

\subsection{Research Content}

This paper mainly developed a new type of insulator string cleaning robot, to achieve the insulator string charged cleaning operations, to solve the current insulator cleaning is facing the main problem. The development of the robot is mainly applicable to the domestic voltage class substation vertical insulator string, has the following advantages:

(1)Mobile flexible and accurate, with a certain ability to cross obstacles 
(2)Low center of gravity, compact structure, smooth work

(3)With automatic cleaning operations, reduce manual operation

(4)The cleaning method is environmentally friendly and does not produce secondary pollution

(5)Support secondary development

This article will focus on the insulator cleaning robot structure design, control system, , a total of four chapters, the main contents are as follows:

The first chapter introduces the background and significance of the study of insulator cleaning robot, and briefly describes the research on the topic of insulator cleaning at home and abroad. Finally, the advantages of the robot are briefly introduced.

The second chapter mainly introduces the structural design of the insulator string cleaning robot. Firstly, the specifications of the insulator strings in the domestic substation are briefly introduced. The design scheme is designed according to the design requirements. The overall structural scheme of the robot is determined by theoretical analysis, and the robot model is finally built. Subsequent simulation experiments.

The third chapter focuses on the control system of the insulator string cleaning robot, and realizes the function of robot automatic cleaning, remote monitoring and control, while leaving the space for secondary development.

In the fourth chapter, the author summarizes the research on insulator cleaning robot, summarizes the shortcomings in the current design, and prospects the future optimization.

\section{Structural Design of Insulator String Cleaning Robot}

\subsection{Section Headings}

Vertical insulator is a pillar insulator, according to the material can be divided into two kinds of ceramic and glass. According to the string index for substation pillar insulator from GB / $\mathrm{T}$ 8287.2-2008 and take the pillar insulator specifications of the common $220 \mathrm{kV}$ substation as an example, table 2-1 shows the insulator parameter index table.

Table 2-1 Insulator Index Table

\begin{tabular}{|l|c|c|c|c|c|c|}
\hline \multirow{2}{*}{ Model } & \multirow{2}{*}{$\begin{array}{c}\text { Height } \\
\text { /mm }\end{array}$} & \multicolumn{4}{|c|}{\begin{tabular}{c} 
Minimum nominal creepage distance \\
The maximum nominal diameter of the insulation \\
\cline { 3 - 6 }
\end{tabular}} & \multicolumn{2}{|c|}{$\begin{array}{c}\text { Thm } \\
\text { C2-550 }\end{array}$} & & & & II & II & IV & 300 \\
C4-550 & $1220 \pm 1$ & 2020 & 2520 & 3150 & 3910 & \\
C6-550 & & & & & & \\
C8-550 & & & & & & \\
\hline
\end{tabular}

According to Table 2-1, the height of the outdoor insulator string of $220 \mathrm{kV}$ substation is $1220 \mathrm{~mm}$ and the maximum nominal diameter is $300 \mathrm{~mm}$. The external shape of the pillar insulator string is concentric and the spacing between the insulators is shown in Fig. 2-1. Figure 2-1 shows the pillar insulator for outdoor plastic. 


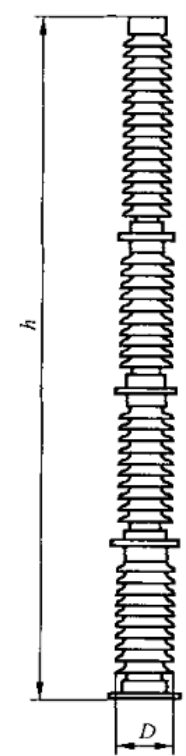

Figure 2-1 Example of outdoor pillow insulator for outdoor rubber

As an automated robot that operates in a high-voltage environment, it is necessary to meet the insulation performance and safety requirements of the control system to avoid paralysis of the equipment and casualties of the staff. According to the electrical safety conditions of high voltage equipment, the required safety distance at $220 \mathrm{kV}$ is $1.6 \mathrm{~m}$. Table $2-2$ is the safe distance for electrical high pressure equipment.

Table 2-2 Safety distance of electrical high pressure equipment

\begin{tabular}{|c|c|c|c|c|c|c|c|}
\hline Voltage level $/ \mathrm{kV}$ & 10 & 35 & 66 & 110 & 220 & 330 & 500 \\
\hline Safe distance $/ \mathrm{m}$ & 0.4 & 0.6 & 0.7 & 1.0 & 1.6 & 2.2 & 3.2 \\
\hline
\end{tabular}

In order to meet the needs of live cleaning, Insulator String Cleaning Robot design using insulating materials (including bolts, brackets, connecting rods, etc.). The robot body components use fiberglass composites, self-designed non-standard parts using MC Nylon, and purchased standard parts using non-glass fibers. Robot motor and high-voltage communication equipment to maintain a safe distance while maximizing the distance, the control circuit board as the bottom of the robot to insulation material. Because dry ice is easy to liquefy the water molecules in the air, droplets and even water mist are generated on the surface of the equipment and on the robot cleaning equipment, reducing the insulation performance of the equipment and the robot. It is necessary to add air drying and filtration systems to the air pressure system to reduce water molecules and impurities in the air and reduce droplets attached to the surface of dry ice. Improve insulation performance as much as possible In the design process.

\subsection{Build the model}

The small insulator string cleaning robot model is based on the above design scheme, used to clean the insulator string model.

Support part of the lift with a stroke of $200 \mathrm{~mm}$ electric putter, the minimum increase is $100 \mathrm{~mm}$, the maximum increase is $300 \mathrm{~mm}$. The movement part of the mechanical claw is made with acrylic plate laser cutting technology, the dimensions are shown in the chart.

\begin{tabular}{|c|c|c|c|c|c|}
\hline & $\begin{array}{c}\text { Outside } \\
\text { diameter }\end{array}$ & Inner diameter & Plate thickness & Plate spacing & angle \\
\hline size $/ \mathrm{mm}$ & 94 & 60 & 5 & 40 & $154^{\circ}$ \\
\hline
\end{tabular}

Rotary brush structure is three tooth imitation gear structure, made with acrylic plate laser cutting, plate diameter is $30 \mathrm{~mm}$, three teeth out of the amount of $30 \mathrm{~mm}$. The total length of the brush is $40 \mathrm{~mm}$, stick to the upper and lower sides of the acrylic plate, set aside $10 \mathrm{~mm}$ out of the plate.

The mechanical stroke of the control mechanical claw is $10 \mathrm{~mm}$. The stroke of the screw that controls the mechanical claw is $200 \mathrm{~mm}$, Its total length is $375 \mathrm{~mm}$. 
The power of the model is rechargeable battery, the battery parameters used in the pump are $12 \mathrm{~V}$, $15000 \mathrm{mAh}$. The battery parameters of the other moving parts are $24 \mathrm{~V}, 3000 \mathrm{mAh}$. The specific parameters are as follows:

The model did not take insulation measures, it used a lot of metal parts, in the production of finished products need to use insulation. The model is completed as shown.

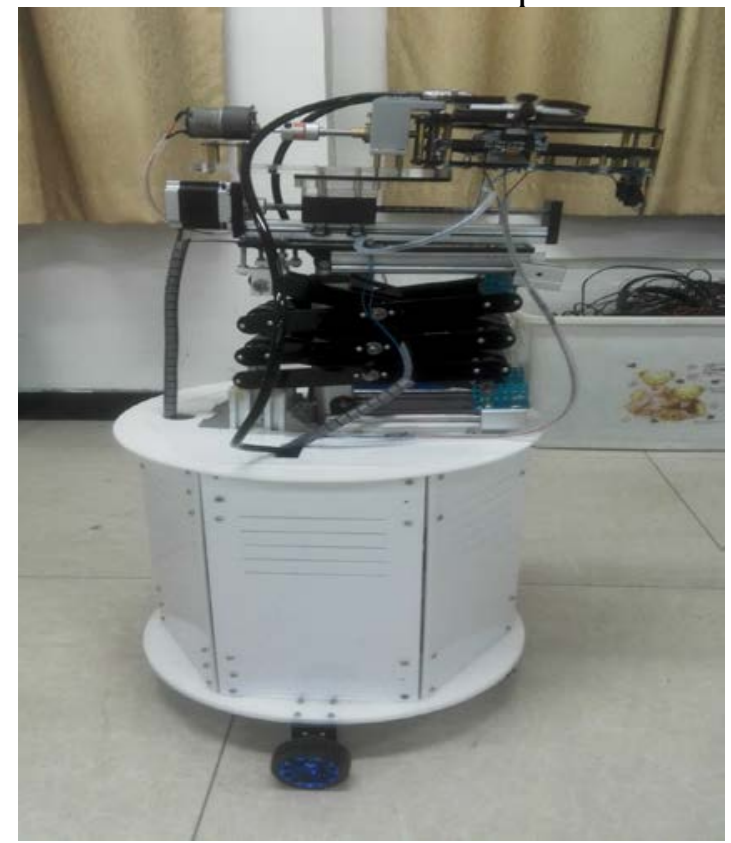

Fig 2-2 Model

\section{Control System of Insulator String Cleaning Robot}

The control system is composed of two parts: the robot control system and the computer wireless control, In order to protect the staff and the robot in the substation complex environment can work safely, The control system mainly uses the computer wireless remote control way. The wireless communication module establishes the online connection between the computer and the Insulator String Cleaning Robot, The computer can real-time access to on-site information by the camera is installed on the robot body ; The robot through the computer to send the action instructions to control the drive module, To complete the end of the tool up, down, forward, back, stretching, condensation and cleaning and other actions to achieve the cleaning of the insulator dirt.

\subsection{Programming}

The main movement of the Insulator String Cleaning Robot including the overall model's forward and backward, turning, electric putter movements, mechanical claw opening and closing and push forward retraction. In order to obtain the optimal control effect, using the STM32 microcontroller for the control board, the Keil5 as the main programming software, and using effective control strategies to avoid or reduce the disturbance, to achieve the overall stability of the system. The specific procedures are as follows:

TIM_TimeBaseInitTypeDef TIM_TimeBaseStructure;

TIM_OCInitTypeDef TIM_OCInitStructure;

GPIO_InitTypeDef GPIO_InitStructure;

RCC_APB2PeriphClockCmd(RCC_APB2Periph_TIM1, ENABLE);

RCC_APB2PeriphClockCmd(RCC_APB2Periph_AFIO, ENABLE);

\section{Conclusions}

(1)Insulator string cleaning robot can achieve the insulator string surface clean full coverage, no dead ends. The robot through a simple mechanical claw open and close the insulator string, with the rotating brush along the mechanical claw movement characteristics, to achieve the insulator string 
surface high quality cleaning effect. Through the manual operation of the robot, greatly reduce the labor force of human labor investment, while avoiding the accidental scrubbing accident occurs, greatly reduce the security risks, and reduce the accident insurance and injury compensation costs. Due to the high cleaning efficiency, the cleaning cycle is short, reducing the time and cost of each cleaning work.

(2)The monitoring system can monitor the pollution accumulation and cleaning quality in real time and feed it back into the robot control system and select the appropriate cleaning force according to the cumulative amount of the surface of the insulator string. The robot can pass the information such as the cleaning quality of each cleaning process through the wireless module Sent to the control system, the user can refer to the information in each cleaning process, in the control system to prepare a suitable local cleaning program. The control system will monitor and control the cleaning of the robot in real time by entering the information. The robot design has the characteristics of automation and targeted.

\section{References}

[1]. Zhi-feng Fan .220kV substation integrated automation system transformation research [D] .Nanchang University .2011: 1-3

[2]. Hong-yu Wang. Causes and Preventive Measures of Pollution Flash Accident [J]. China High-Tech Enterprise .2009,5: 101-102

[3]. Jing-an Ye. EHV live working robotic cleaning device Optimized Design and Research [D] Shanghai Jiaotong University 2008: 1-5

[4]. Yi-zhuang Huang. Electromagnetic Compatibility of Intelligent Electronic Equipment in Substation [J]. Power System Protection and Control. 2008 (15)

[5]. Rong Hu. Experimental Study on Voltage Characteristics of Transient Shells of UHV Gas Insulated Switchgear [J]. Proceeding of the CSEE (in Chinese), 2004 (29) 